\title{
Los mamíferos marinos en la costa central de Oaxaca
}

\author{
Marine mammals along the central coast of Oaxaca
}

\author{
Juan Meraz ${ }^{1 *}$ y Víctor Manuel Sánchez-Díaz ${ }^{2}$ \\ ${ }^{1}$ Department of Evolutionary and Environmental Biology. Graham Kerr Building, University of Glasgow, United Kingdom \\ ${ }^{2}$ Biología Marina, Universidad del Mar, Puerto Angel, Oaxaca, México \\ *Correspondencia: j.meraz-hernando.1@research.gla.ac.uk
}

\begin{abstract}
Resumen. Con la finalidad de conocer las especies de mamíferos marinos que se encuentran en la costa central de Oaxaca, se elaboró un listado comentando las características de los registros obtenidos. Se incluyeron las observaciones hechas a lo largo de 30 recorridos por mar, entre junio de 1999 y enero de 2004, realizadas entre las bahías de Huatulco y la playa de La Escobilla. Adicionalmente se incluyeron registros de animales varados, así como observaciones realizadas desde la costa. Se presentan comentarios sobre el registro de 8 especies en la zona, incluyendo las localidades y fechas de los avistamientos. Stenella attenuata es la especie más abundante, y la zona de Zipolite-Isla Roca Blanca la localidad con el mayor número de avistamientos. Dado el crecimiento turístico del lugar, es importante contar con este tipo de registros ya que es poco lo que se sabe sobre estas especies en esta porción de la costa oaxaqueña.
\end{abstract}

Palabras clave: Oaxaca, Tursiops truncatus, Stenella attenuata, S. longirostris, Feresa attenuata, Pseudorca crassidens, Orcinus orca, Megaptera novaeangliae, Zalophus californianus.

\begin{abstract}
With the aim of documenting the species of marine mammals that occur along the central coast of Oaxaca, a list with the characteristics of the records was elaborated based on observations made throughout 30 surveys from Huatulco Bays to La Escobilla beach between June 1999 and January 2004. Additional records from stranded animals are included, as well observations from the coast. Comments on 8 species are presented, including localities and dates of sightings. Stenella attenuata is the most abundant species. Zipolite-Roca Blanca island was the locality with the highest number of sightings. This records are important to promote conservation due growth of tourism in the area, given that Oaxaca coast is poorly known regarding marine mammals.
\end{abstract}

Key words: Oaxaca, Tursiops truncatus, Stenella attenuata, S. longirostris, Feresa attenuata, Pseudorca crassidens, Orcinus orca, Megaptera novaeangliae, Zalophus californianus.

\section{Introducción}

El Pacífico sur mexicano comprende la porción tropical que va desde Cabo Corrientes, Jalisco, hasta la frontera con Guatemala. Presenta una riqueza específica de mamíferos marinos estimada en 18 especies, la cual supera la del golfo de México y mar Caribe, pero es menor que la del Pacífico norte de México y el golfo de California (Aguayo-Lobo et al., 1990). Respecto a mamíferos marinos, los estudios que se han hecho en la costa del estado de Oaxaca han sido pobres, y sólo existen informes previos sobre registros muy locales o anecdóticos. Este trabajo, por tanto, es el primer registro sistemático de las especies presentes. Su utilidad es relevante para conocer la fauna mastozoológica marina de México (Aurioles-Gamboa, 1993), básicamente porque complementa la información existente. Las listas de

Recibido: 21 septiembre 2006; aceptado: 12 septiembre 2007 riqueza específica incluyen tanto las especies registradas como aquellas cuya presencia se considera probable. El presente listado es de particular importancia porque es resultado de la búsqueda de estos organismos mediante recorridos periódicos por mar a lo largo de un año.

En las costas de Oaxaca los mamíferos marinos están representados exclusivamente por cetáceos. Se ha considerado la presencia de las especies: Tursiops truncatus, Stenella attenuata, Stenella longirostris, Feresa attenuata, Pseudorca crassidens, Grampus griseus, Globicephala macrorhynchus, y Megaptera novaeangliae (Salinas y Ladrón de Guevara, 1993). Se suponía que la ballena jorobada se distribuía en México desde la costa occidental de Baja California hasta las costas de Nayarit y Jalisco (Aguayo-Lobo et al., 1984) y registros posteriores la consideran visitante invernal en la porción tropical del Pacífico mexicano (Gallo-Reynoso et al., 1986). Actualmente se reconoce que una subpoblación migra 
hasta Centroamérica, llegando a Costa Rica y Panamá (Steiger et al, 1991).

Registros adicionales de otras especies de cetáceos en Oaxaca incluyen a Orcinus orca (Sánchez-Díaz y Meraz, 2001), Physeter macrocephalus y Ziphius cavirostris (Pérez-Bouchez y Gordillo-Morales, 2002). Otros registros confiables corresponden a 8 individuos de T. truncatus en Escobilla, en septiembre de 1978, 6 de Physeter macrocephalus 12 millas náuticas mar adentro en Huatulco, en marzo de 1990, y 16 individuos de Stenella attenuata en las inmediaciones de Playa del Amor, en marzo de 1990 (Gallo-Reynoso, 2007, comunicación personal).

Para los mamíferos marinos del orden Carnivora en el estado de Oaxaca, se tienen registros extraordinarios de Zalophus californianus en las bahías de Huatulco (Meraz, 2003) y en las inmediaciones de Puerto Ángel (GalloReynoso y Solórzano-Velasco, 1991), que se suman a varios registros previos en el Pacífico tropical mexicano en Acapulco, Guerrero, y aguas mar adentro de Puerto Madero, Chiapas (Gallo-Reynoso y Ortega-Ojeda, 1986; GalloReynoso y Solórzano-Velasco, 1991).

El objetivo del presente trabajo es ofrecer un panorama amplio sobre la riqueza de especies de mamíferos marinos en Oaxaca, todas ellas bajo algún estatus de protección en México (NOM-059-ECOL-2001), y aportar información acerca de la distribución, estacionalidad y conductas observadas, así como de las condiciones en que se obtuvieron los registros.

\section{Materiales y métodos}

La costa de Oaxaca (Fig. 1) se encuentra en los límites de 2 regiones oceanográficas: la mexicana que comprende desde cabo San Lucas, Baja California Sur, hasta el límite con el golfo de Tehuantepec, caracterizada por corrientes débiles y variables que en invierno tienen una dirección hacia el sureste y en verano hacia el noroeste, y la centroamericana que comprende desde el golfo de Tehuantepec hasta Panamá, siendo su principal característica la influencia de vientos tanto del Pacífico como del Atlántico (SEMARNAP, 1998). El patrón de movimiento oceanográfico en la zona está determinado por la corriente de California, que es fría con bajas salinidades y un alto contenido de oxígeno disuelto, y la nor-ecuatorial, caliente con altas salinidades y bajo contenido de oxígeno disuelto (Pacheco-Sandoval, 1991). Sin embargo, de manera estacional la zona se ve afectada por la corriente más costera de Costa Rica, que en junio y julio sigue la costa centroamericana hacia el norte hasta cabo Corrientes. En agosto se aleja de la costa al pasar el golfo de Tehuantepec, estando ausente de enero a marzo en la zona, y desarrollándose nuevamente en abril y mayo (Pacheco-Sandoval, 1991).

En condiciones normales la zona presenta una temperatura que varía de 25 a $30^{\circ} \mathrm{C}$ en los primeros $30 \mathrm{~m}$ de profundidad, estando al sur de la isoterma de $\operatorname{los} 33^{\circ} \mathrm{C}$ durante la mayor parte del año (Vázquez-Gutiérrez et al., 1998). La termoclina es somera y permanente, presentándose la capa de mezcla a los 20 m (Pacheco-Sandoval, 1991).

El área de estudio del presente trabajo comprendió la porción central de la costa de Oaxaca (Fig. 1), ubicada entre la playa La Escobilla ( $\left(15^{\circ} 43^{\prime} \mathrm{N}\right.$ y $\left.96^{\circ} 45^{\prime} \mathrm{O}\right)$ al noroeste, y la isla Montosa $\left(15^{\circ} 45^{\prime} \mathrm{N}\right.$ y $\left.96^{\circ} 04^{\prime} \mathrm{O}\right)$ al sureste. Dicha costa está caracterizada por playas rocosas, enclavadas en extensas playas arenosas en la línea de costa. La zona no presenta lagunas costeras importantes (salvo un par de esteros de boca efímera). Los sistemas lagunares importantes se encuentran en Chacahua-Pastoría al noroeste de la costa del estado. En las inmediaciones de la zona de estudio existen varias islas de tamaño considerable, concentradas principalmente a lo largo de las bahías de Huatulco (Meraz, 2001). Las islas de mayor tamaño presentes en la zona son Cacaluta, San Agustín y Montosa. Existen también otras 11 islas menores a una hectárea (SEMARNAP, 1998).

Se realizaron 20 recorridos, entre octubre del 2000 y octubre del 2001, a bordo de una embarcación de fibra de vidrio de $6.7 \mathrm{~m}$ de eslora, equipada con un motor fuera de borda de 45 HP. Estos recorridos se realizaron en 2 días consecutivos por cada mes. El primer día se recorrió desde la isla Roca Blanca, ubicada frente a la playa Zipolite, hasta la bahía de San Agustín en Huatulco (recorriendo $67 \mathrm{~km}$ ). El segundo día se recorrió desde la bahía de Puerto Ángel hasta la isla La Montosa, ubicada en la bahía de Tangolunda, Huatulco (recorriendo 100 km). Es importante mencionar que los recorridos de ida, partiendo

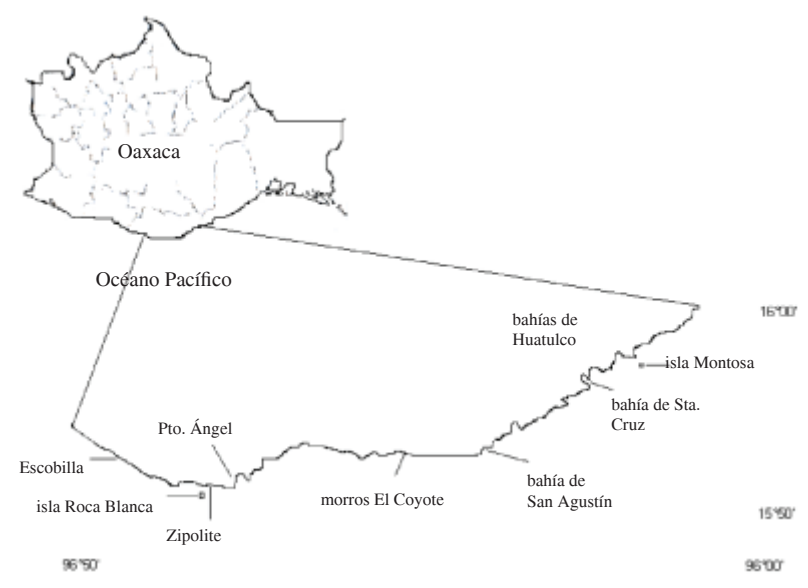

Figura 1. Porción central de la costa de Oaxaca donde se obtuvieron los registros. 
de Puerto Ángel, se realizaron a no más de $300 \mathrm{~m}$ de la línea de costa, mientras que los recorridos de regreso se realizaron más alejados de ella, entre 1000 y 2000 m. El grupo estuvo formado por un patrón motorista, un auxiliar y 3 observadores, uno de ellos, se colocó a, a estribor, el otro a babor y el tercero en la proa de la embarción. A fin de detectar la presencia de mamíferos marinos se utilizaron binoculares $10 \times 50$.

Se realizaron 10 recorridos matutinos adicionales, de distancias y trayectorias variables, entre la playa La Escobilla y las bahías de Huatulco, entre junio de 1999 y enero del 2004.

Adicionalmente, se tomaron registros a partir de organismos varados en las inmediaciones de la playa de Zipolite, así como en las bahías de Huatulco. Finalmente, se incluyen observaciones realizadas desde la playa Escobilla, que es la principal zona de anidación de la tortuga golfina Lepidochelys olivacea (Márquez, 2000).

Para la elaboración del listado se empleó la Guía FAO (Jefferson y Leatherwood, 1995) y la guía de la Comisión Interamericana del Atún Tropical (Leatherwood et al., 1988).

\section{Resultados}

Todos los recorridos por mar se realizaron en la mañana, a partir de las 9:00, en condiciones de mar en calma, con excepción del realizado durante el mes de marzo del 2001 donde se presentó mar agitado en la porción sureste del área de estudio (que corresponde a las bahías de Huatulco). Salvo en 2 ocasiones durante los meses de julio y agosto del 2001, en que se presentó cielo nublado y ligera llovizna, el resto de los recorridos se realizó con clima soleado. El esfuerzo de observación varió entre 5 y 6 horas, dependiendo tanto de las condiciones ambientales como del tiempo destinado a las propias observaciones.

Se obtuvo el registro de 8 especies de mamíferos marinos en la zona (Cuadro 1), 7 especies del orden Cetacea y una especie del orden Carnivora. La especie con los mayores valores de abundancia fue $S$. attenuata (hasta 60 individuos por recorrido), seguida de $S$. longirostris y T. truncatus (Cuadro 2). Los mayores valores del índice de abundancia relativa (número de individuos observados por kilómetro recorrido), fueron para $S$. attenuata en los meses de enero, mayo y julio (Cuadro 2). Las especies con mayor presencia, que podrían considerarse como las dominantes en la zona, fueron $S$. attenuata que se observó en casi todos los recorridos y T. truncatus.

Hubo un registro visual de un rorcual, que por la brevedad de la observación (se observó a lo lejos por unos 10 segundos y se sumergió) no pudo identificadorse la especie. Se supone que en la zona puede encontrarse el rorcual tropical (Balaenoptera edeni), y se sabe de la presencia de la ballena azul (B. musculus), dado que recientemente se registró el varamiento de un ejemplar de esta especie en Barra de Navidad, en las inmediaciones de Puerto Escondido.

Durante los meses de enero, mayo, noviembre y diciembre del 2001 se presentaron las mayores frecuencias de avistamientos. Por el contrario, en diciembre del año 2000 y junio del 2001 se registraron las frecuencias más bajas (Fig. 2).

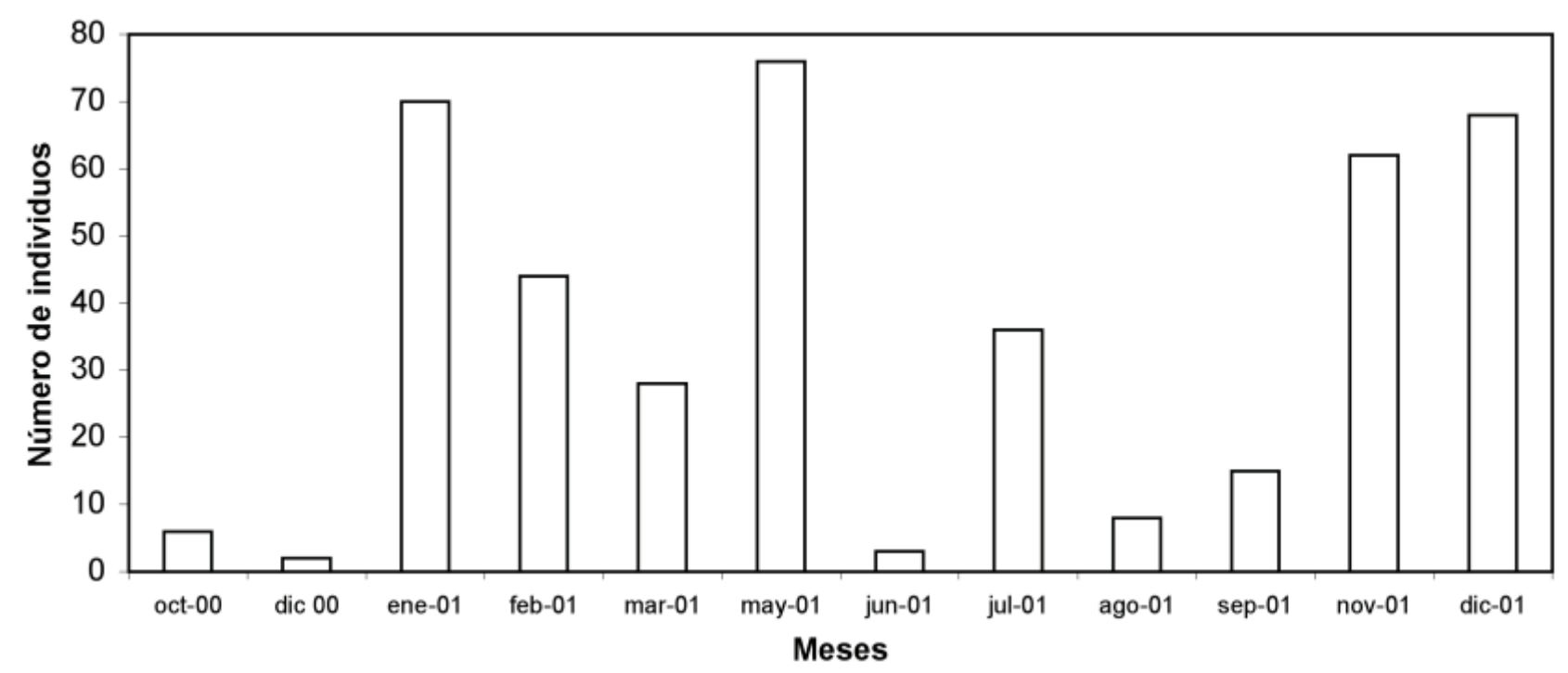

Figura 2. Frecuencia de las observaciones por mes para todas las especies, considerando los registros obtenidos durante los recorridos por mar. 
Cuadro 1.Relación de las especies de mamíferos marinos registrados en la costa central de Oaxaca y su temporalidad. Se incluyen tanto los registros realizados durante los muestreos por mar, como aquellos obtenidos desde la costa y por los varamientos

\begin{tabular}{|c|c|c|c|c|c|c|c|c|c|c|c|c|}
\hline Especie & Ene & $\mathrm{Feb}$ & Mar & $A b r$ & May & Jun & $J u l$ & Ago & Sep & $O c t$ & Nov & Dic \\
\hline Stenella attenuata & $\mathrm{x}$ & $\mathrm{x}$ & $\mathrm{x}$ & $\mathrm{x}$ & $\mathrm{x}$ & & $\mathrm{x}$ & $\mathrm{x}$ & $\mathrm{x}$ & & $\mathrm{x}$ & $\mathrm{x}$ \\
\hline Stenella longirostris & & & & & $\mathrm{x}$ & & & & & & & \\
\hline Tursiops truncatus & $\mathrm{x}$ & & $\mathrm{x}$ & & $\mathrm{x}$ & & & $\mathrm{x}$ & & & & \\
\hline Orcinus orca & & & & & & & & & & $\mathrm{x}$ & & $\mathrm{x}$ \\
\hline Pseudorca crassidens & & & & & & $\mathrm{x}$ & & & & & & \\
\hline Feresa attenuata & & & & & & & & & & & & $\mathrm{x}$ \\
\hline Megaptera novaeangliae & & $\mathrm{x}$ & & & & & & & & $\mathrm{x}$ & & \\
\hline Zalophus californianus & & & & & $\mathrm{x}$ & $\mathrm{x}$ & & & & & & \\
\hline
\end{tabular}

Cuadro 2. Abundancias por mes de las principales especies de cetáceos registradas durante los recorridos por mar más representativos. Se indica el número de individuos registrados, así como el valor del índice de abundancia relativo (número de individuos observados por kilómetro recorrido) en cursivas

\begin{tabular}{|c|c|c|c|c|c|c|c|c|c|}
\hline Especie & Oct 00 & Ene 01 & $\mathrm{Feb} 01$ & Feb 01 & Mar 01 & May 01 & May 01 & Jul 01 & Ago 01 \\
\hline Megaptera novaeangliae & $\frac{1}{0.014}$ & & $\frac{1}{0.010}$ & & & & & & \\
\hline Stenella attenuata & & $\begin{array}{c}60 \\
0.895\end{array}$ & $\begin{array}{c}34 \\
0.340\end{array}$ & $\stackrel{5}{0.074}$ & $\begin{array}{c}25 \\
0.250\end{array}$ & $\begin{array}{c}7 \\
0.104\end{array}$ & $\begin{array}{c}40 \\
0.597\end{array}$ & $\begin{array}{c}36 \\
0.537\end{array}$ & $\stackrel{4}{0.059}$ \\
\hline Stenella longirostris & & & & & & & $\begin{array}{c}10 \\
0.149\end{array}$ & & \\
\hline Orcinus orca & $\begin{array}{c}5 \\
0.074\end{array}$ & & & & & & & & \\
\hline \multicolumn{10}{|l|}{ Feresa attenuata } \\
\hline Tursiups truncatus & & $\begin{array}{c}10 \\
0.149\end{array}$ & & & $\begin{array}{c}3 \\
0.030\end{array}$ & $\begin{array}{c}1 \\
0.014\end{array}$ & $\begin{array}{c}12 \\
0.179\end{array}$ & & $\begin{array}{c}4 \\
0.059\end{array}$ \\
\hline
\end{tabular}

Las zonas que presentaron los mayores porcentajes de avistamiento para todos los individuos de todas las especies, fueron Zipolite-isla Roca Blanca con el 18\%, Morros-playa el Coyote con el $10.6 \%$ y bahía de Santa Cruz con el $8.2 \%$. Por el contrario, en la isla Montosa no se obtuvo registro alguno en las 10 visitas que se hicieron por mar. A continuación se presentan las especies encontradas con comentarios sobre sus registros en la zona.

Stenella attenuata (delfín manchado o estenela moteada del Pacífico).
La forma general del cuerpo, esbelto e hidrodinámico, es muy semejante a la del delfín común; llegan a medir $2.6 \mathrm{~m}$ de longitud y pesan hasta $120 \mathrm{~kg}$ (Jefferson y Leatherwood, 1995). Presentan rostro largo y delgado separado del melón por un pliegue evidente (Leatherwood et al., 1988). La distribución de los delfines manchados incluye las aguas tropicales de todo el mundo, y se reconocen 4 stocks en la parte este y central del océano Pacífico (Dizon et al., 1994).

Es la especie que con mayor frecuencia se ve en la zona (más del 84\% de los individuos observados correspondieron a ésta), pudiéndose observar durante todo el año en manadas 
Cuadro 2. Continúa

\begin{tabular}{|c|c|c|c|c|c|}
\hline Especie & Sep 01 & Nov 01 & Nov 01 & Dic 01 & Dic 01 \\
\hline M. $n$. & & & & 2 & \\
\hline S. $a$. & $\begin{array}{l}13 \\
0.130\end{array}$ & $\begin{array}{l}20 \\
0.298\end{array}$ & $\begin{array}{l}42 \\
0.420\end{array}$ & $\begin{array}{l}30 \\
0.447\end{array}$ & $\begin{array}{l}30 \\
0.300\end{array}$ \\
\hline \multicolumn{6}{|l|}{ S. $l}$. \\
\hline \multicolumn{6}{|l|}{ O. $о}$. \\
\hline F. $a$. & & & & & $\begin{array}{l}6 \\
0.060\end{array}$ \\
\hline T.t. & & & & & \\
\hline
\end{tabular}

de hasta 100 individuos o más, a lo largo del área recorrida. A menudo se les observó acompañadas por un número notablemente menor de $S$. longirostris y $T$. truncatus. Comúnmente se les encontró transitando o alimentándose en subgrupos de 5-20 individuos.

Aunque no se observaron individuos reproduciéndose, fue posible registrar crías (menores a $1 \mathrm{~m}$ de longitud), lo que supone su reproducción en la zona.

Se tienen registros de un par de ejemplares varados, en Zipolite y en la bahía de Chahué (Huatulco), y es común obtenerreferencias de los pescadores locales sobre numerosos ejemplares varados a lo largo de la costa oaxaqueña. No se descarta la posibilidad de que algunos ejemplares se cacen con el fin de ser utilizados como carnada para la pesca de tiburón, con cimbra o palangre, como sucede en otras partes de México.

Stenella longirostris orientalis (delfín tornillo o estenela giradora).

Presenta un cuerpo esbelto y pequeño, con rostro extremadamente largo y delgado, y cabeza muy angosta a nivel del ápice del melón (Leatherwood et al., 1988; Perrin, 1998). Posee una aleta triangular erecta, siendo ésta la mejor característica para reconocerla en el campo. Existen 2 subespecies similares, $S$. $l$. orientalis y $S$. $l$. centroamericana; la segunda, ligeramente más grande en talla, que pueden encontrarse en el Pacífico tropical oriental. De acuerdo a la distribución registrada para ambas subespecies (Perrin, 1998), es de suponerse que $S$. $l$. orientalis es la que está presente en la costa de Oaxaca, ya que la subespecie centroamericana se ha registrado más al sur, en aguas de Costa Rica.

Su distribución es casi idéntica a la de $S$. attenuata, por lo cual, no fue raro encontrar ambas especies asociadas (Leatherwood et al., 1988). Durante los recorridos, se observaron 10 ejemplares asociados con 13 individuos de $T$. truncatus y con 40 de $S$. attenuata durante el mes de mayo del 2001.

En marzo del 2004 se registró el varamiento de un macho adulto de $184 \mathrm{~cm}$ de longitud, en Playa del Amor, en las inmediaciones de Zipolite. Aunque se han registrado este tipo de eventos para $S$. longirostris, no son muy comunes (Odell, 1987). Debido a las marcas en el cuerpo, se pudo determinar que la causa de su muerte fue el enmallamiento en una red pesquera. El ejemplar fue colectado y depositado en el Laboratorio de Oceanografía Biológica de la UMAR. Por razones de manejo deficiente durante su traslado y preparación, sólo se conservan partes del cráneo y de la columna vertebral. Salvo este ejemplar varado, esta especie únicamente se observó en las bahías de Huatulco y en los morros El Coyote durante la primavera y el verano.

Tursiops truncatus (tonina, tursión o delfín nariz de botella).

Es un delfín grande y robusto, con una talla máxima de $4 \mathrm{~m}$ y un peso de $450 \mathrm{~kg}$ (Leatherwood et al., 1988). Presenta un rostro ancho y corto, así como un melón bien demarcado por un pliegue (Jefferson y Leatherwood, 1995). Su prominente aleta dorsal se encuentra situada en el centro del lomo.

Se considera cosmopolita en aguas cálido-templadas, siendo una especie de hábitos alimentarios oportunistas (Delgado, 1997) que incluyen ataques en forma coordinada entre varios individuos a cardúmenes de peces, o bien, se le puede encontrar alimentándose de manera solitaria (Leatherwood et al., 1988).

Esta fue la especie con el segundo mayor porcentaje de avistamientos $(7.7 \%)$, y se observó de enero a agosto en grupos pequeños (entre 2 y 13 organismos), siempre asociada con $S$. attenuata. Estas asociaciones entre T. truncatus y delfines del género Stenella han sido previamente documentadas en México (Delgado, 1997).

\section{Orcinus orca (orca ).}

Esta es una de las especies de odontocetos más grande (Leatherwood et al., 1988). Los machos crecen hasta los 9.5 metros de longitud y llegan a pesar 8 toneladas; las hembras son significativamente más pequeñas.

Las características más reconocibles de esta especie son el tamaño corporal, la longitud de su aleta dorsal de forma triangular en los machos y falcada en las hembras, y su coloración negra con vientre y mancha supraocular blancos (Nowak y Paradiso 1983; Heyning y Dahlheim, 1988; 
Leatherwood et al., 1988; Jefferson y Leatherwood, 1995; Raymond 1996). El rostro es de forma roma (Jefferson y Leatherwood, 1995), corto y poco definido (Nowak y Paradiso, 1983).

Orcinus orca se puede encontrar en todos los océanos del mundo, y es más común en aguas templadas cercanas a las costas (Nowak y Paradiso, 1983; Leatherwood et al., 1988; Jefferson y Leatherwood, 1995). Ampliamente distribuida en el Pacífico nororiental, desde el mar de Chukchi hasta el Ecuador (Leatherwood et al., 1988).

Se considera que en el Pacífico centro-oriental las manadas de orcas pueden estar compuestas de 2 a 4, de 55 (Leatherwood et al., 1988; Jefferson y Leatherwood, 1995), e incluso hasta 250 ejemplares (Nowak y Paradiso, 1983).

En octubre del año 2000 se observó un grupo de 5 individuos en las inmediaciones de la isla Roca Blanca. Se identificaron 3 hembras, 1 macho y 1 juvenil que depredaron a una tortuga laúd (Dermochelys coriacea), mientras desplegaban la conducta kerplunking (golpeteo de la superficie del agua con los lóbulos caudales durante la alimentación; Connor et al., 2000) ante la cercanía de nuestra embarcación (Sánchez-Díaz y Meraz, 2001).

\section{Pseudorca crassidens (orca falsa).}

Presenta cuerpo alargado y esbelto (Jefferson y Leatherwood, 1995); cabeza estrecha que se reduce a partir del orificio respiratorio hacia la parte anterior; la frente es sobresaliente, por lo cual no se observa su separación con el rostro (Nowak y Paradiso, 1983; Leatherwood et al., 1988 ; Jefferson y Leatherwood, 1995). Estos organismos tienen una aleta dorsal falcada y redondeada, situada por detrás del punto medio del dorso (Nowak y Paradiso, 1983; Leatherwood et al., 1988). La coloración va de gris obscura (Jefferson y Leatherwood, 1995), a negro (Nowak y Paradiso, 1983; Leatherwood et al., 1988), a excepción de una porción gris clara en forma de ancla, situada en el pecho entre las aletas pectorales. Los adultos llegan a medir $6 \mathrm{~m} \mathrm{y}$ pesar 1.3 toneladas (Nowak y Paradiso, 1983).

$\mathrm{Su}$ abundancia es poco conocida en México (AuriolesGamboa, 1993; Salinas y Ladrón de Guevara, 1993). Son organismos muy sociables, por lo cual pueden llegar a formar grupos o manadas que comprenden hasta 500 individuos; sin embargo, es común encontrar grupos de 2 a 60 individuos (Nowak y Paradiso, 1983; Leatherwood et al., 1988). Su distribución se extiende por las aguas cálidotempladas de ambos hemisferios (Jefferson y Leatherwood, 1995).

En la costa de Oaxaca se dio el primer registro para el estado de un ejemplar varado de esta especie: el cadáver de un macho adulto de $525 \mathrm{~cm}$ de longitud desde la punta del rostro hasta la escotadura de los lóbulos de la aleta caudal que se encontró en las inmediaciones de Playa del Amor
(Meraz y Becerril-Morales, 2004). Esto coincide con el lugar donde también varó el ejemplar de $S$. longirostris, del que se hizo referencia anteriormente.

Feresa attenuata (orca pigmea).

Es un cetáceo poco común, que frecuentemente se confunde con la falsa orca y el calderón Globicephala macrorhynchus (Jefferson y Leatherwood, 1995). Presenta un cuerpo delgado que alcanza $2.7 \mathrm{~m}$ de longitud en los adultos, y una cabeza redondeada carente de rostro (Nowak y Paradiso, 1983).

Seconsideraquesualetadorsal es unadelascaracterísticas diagnósticas más importantes para diferenciarlas de la orca falsa y el calderón, pues es prominente, falcada, y situada cerca de la mitad del dorso (Leatherwood et al., 1988).

La coloración es gris oscuro o negro y presenta un manto dorsal no muy definido de una tonalidad ligeramente diferente. Usualmente presenta 2 áreas blancas, una localizada en la región ventral posterior al ombligo y otra alrededor de la boca (Leatherwood et al., 1988; Raymond, 1996) . Son organismos cosmopolitas presentes en aguas oceánicas tropicales y subtropicales (Leatherwood et al., 1988; Jefferson y Leatherwood, 1995), que forman manadas hasta de un centenar de individuos (Leatherwood et al., 1988). En las costas oaxaqueñas se había considerado su presencia como probable (Aurioles-Gamboa, 1993); Salinas y Ladrón de Guevara (1993), la consideran una especie presente en Oaxaca. En diciembre del 2001 se pudieron observar 6 individuos en tránsito, en las inmediaciones de los Morros el Coyote. El grupo se mostró esquivo, sumergiéndose rápidamente para alejarse de la embarcación. En noviembre del 2003 se observó un grupo de 3 individuos nadando a escasos $10 \mathrm{~m}$ de la costa en la playa La Escobilla. Este registro se asoció con la presencia de varias hembras adultas de tortuga golfina (L. olivacea), que entraban al mar, durante el amanecer, después de haber ovopositado en la playa. Por ello, podría considerarse la probabilidad de que estas tortugas sean parte de la dieta de F. attenuata.

Megaptera novaeangliae (rorcual jorobado o ballena jorobada).

Presenta un cuerpo robusto de $15 \mathrm{~m}$ de largo y 41 toneladas de peso; las hembras son ligeramente más grandes que los machos (Leatherwood et al., 1988).

La coloración varía de negro a gris oscuro con manchas blancas irregulares en la garganta, costados y abdomen. Las aletas pectorales extremadamente largas son de color blanco en la parte ventral. La aleta dorsal es pequeña y de forma variada, desde triangular hasta falcada (Jefferson y Leatherwood, 1995).

Son catalogados como organismos sociables, por lo cual no es raro que se les encuentre en grupos de 7 a 
10 individuos.

Existen 4 sub-regiones de distribución en México: la costa sur de Baja California, el norte del golfo de California (incluyendo las grandes islas), la costa continental del Pacifico y el archipiélago de las Revillagigedo, siendo poco comunes en las costas del Pacifico sur de nuestro país (Aurioles-Gamboa, 1993; Gendron, 1993). No obstante, cada vez con más frecuencia, se han registrado individuos desde Jalisco hasta Oaxaca (Aguayo-Lobo et al., 1984) así como en Guerrero (Gallo-Reynoso et al., 1986). Se considera que las migraciones de $M$. novaeangliae abarcan todo el litoral del Pacífico mexicano, ya que se han registrado individuos migrando desde las costas de California hasta las aguas cercanas a Costa Rica (Steiger et al., 1991). Los factores más importantes en la ampliación de su distribución son las condiciones físico-químicas del agua (principalmente temperatura) que pueden limitar o favorecer sus desplazamientos (Aurioles-Gamboa, 1993). En octubre del 2000 se observó un individuo solitario, aproximadamente a $2 \mathrm{~km}$ de la costa, al suroeste de las bahías de Huatulco. Por otra parte, en febrero del 2001 se observó en las inmediaciones de Puerto Ángel una pareja que transitaba en dirección noroeste; en diciembre del 2001 se registró otra pareja en las bahías de Huatulco, con dirección sureste. Ambas parejas viajaban muy cerca de la costa. En enero del 2004 se observó un grupo de 3 individuos nadando frente a la playa de Escobilla, con dirección al sureste, a escasos 20 m de la costa.

Es importante mencionar que los avistamientos de estas ballenas por los pescadores y prestadores de servicios turísticos son comunes a lo largo del corredor turístico Huatulco-Puerto Escondido durante el invierno.

Zalophus californianus californianus (lobo marino de California).

Es el pinnípedo de más amplia distribución en México (Aurioles-Gamboa, 1993), y abarca todo el Pacífico norte y el Golfo de California (Aurioles-Gamboa et al., 1983). Los puntos más sureños donde se han registrado individuos de esta especie son las islas Marías (Hall, 1981; Odel, 1981), las costas de Guerrero (Gallo-Reynoso y OrtegaOjeda, 1986; Salinas y Ladrón de Guevara, 1993), Oaxaca (Meraz, 2003) y Chiapas (Gallo-Reynoso y SolórzanoVelasco, 1991).

En marzo del 2001 se observó un ejemplar de $Z$. californianus nadando en la Playa La Ventanilla, a escasos $5 \mathrm{~km}$ al noroeste de Zipolite. El mes siguiente se observó un individuo (probablemente el mismo) en la bahía de Riscalillo, aledaña a la de San Agustín (Meraz, 2003). Durante el mes de mayo se realizaron 4 visitas a la bahía de Riscalillo para observar el animal, registrándose por última ocasión el 1 de junio.
Es un registro extraordinario para la zona y no podría decirse que esta especie es visitante estacional de la costa de Oaxaca. Por ello, se considera que Z. californianus es una especie ocasional para la zona.

\section{Discusión}

La costa de Oaxaca presenta varias islas pequeñas, morros y bahías que pueden servir de refugio a algunas especies de pequeños cetáceos. Algunas lagunas costeras y ríos aportan nutrientes a la costa que, aunado a la presencia de surgencias costeras (especialmente en la porción sur del estado), son un factor importante para mantener una elevada productividad.

A pesar de que los mamíferos marinos están pobremente representados en la costa de Oaxaca, es común encontrar algunas especies (principalmente $S$. attenuata) a lo largo del año. Esto, como ya se dijo, hace suponer que es una especie residente en la zona. Situación semejante podrían presentar $T$. truncatus y $S$. longirostris, aunque con abundancias menores.

Es importante reconocer que faltaron por realizarse recorridos más alejados de la costa, lo cual incrementaría la lista de especies registradas.

Para el caso de los grandes delfínidos (O. orca, $P$. crassidens y $F$. attenuata) la zona presenta alimento disponible en grandes cantidades, principalmente porque existen importantes playas de anidación en masa de tortugas marinas. Por ello es importante considerar que estas especies, así como otras con hábitos alimenticios semejantes, pudieran estar mejor representadas en las inmediaciones de dichas playas durante el proceso de anidación de las tortugas marinas. De hecho, O orca y $F$. attenuata se registraron en los meses de octubre y diciembre, cuando se presentan las arribadas importantes de L. olivacea (Casas-Andreu, 1978; Ávila Barrientos, com. pers. 2005). Además, en mar abierto se puede encontrar atún de aleta amarilla y otros escómbridos que son parte de la dieta de estos delfínidos.

Si bien los registros ocasionales de Z. californianus al sur de bahía de Banderas (en Jalisco y Nayarit) se consideran de poco significado en la distribución habitual de la especie (Aurioles-Gamboa y García-Rivas, 2005), pueden significar esfuerzos individuales para buscar nuevas áreas de distribución. Podría tratarse de individuos jóvenes y subadultos en fase de alimentación y crecimiento que regresan a las zonas reproductivas para competir por hembras y territorios. La competencia por los espacios y los recursos en las loberas del sur del golfo de California, pudiera presionar a algunos individuos a buscar nuevos sitios de distribución, lo cual se explicaría con la teoría de 
la distribución libre ideal (Sutherland, 1983).

De todas las localidades visitadas, isla Montosa fue la única donde nunca se registró animal alguno. Ello pudiera tener relación con las actividades turísticas, paseos en lancha principalmente, que son intensas ahí, o con el simple hecho de que no sea un área de importancia para estos organismos. Por otra parte, existen prestadores de servicios turísticos que llevan turistas a ver delfines, tanto en las bahías de Huatulco como en Puerto Ángel y Mazunte (al oeste de Zipolite). Es importante tomar esto en consideración, dado que se espera un notable incremento en las actividades turísticas en la costa de Oaxaca.

Las actividades pesqueras en la costa de Oaxaca son de tipo ribereño. Las zonas de pesca de camarón, donde se emplean embarcaciones mayores, se localizan en la porción sur de la costa del estado, teniendo como base el puerto de Salina Cruz. Es importante realizar observaciones de cetáceos desde estas embarcaciones en la región, debido a que muchos delfines suelen asociarse con esta actividad pesquera (Delgado, 1997).

La evaluación de la riqueza de la fauna mastozoológica en Oaxaca, así como el seguimiento de sus poblaciones, es una tarea que debe desarrollarse de manera constante. De esta forma se podrá conocer la ecología de estas especies en la zona e incrementar el conocimiento de los mamíferos marinos en México.

\section{Agradecimientos}

Los autores agradecen a J. P. Gallo Reynoso por compartir sus observaciones con nosotros, así como a 2 revisores anónimos, cuyas sugerencias mejoraron sustancialmente este trabajo. A Betzabeth GonzálezBravo por su apoyo en los recorridos por el mar. A Hugo, Heladio, Andrés y Leo, motoristas de la Universidad del Mar (UMAR).

\section{Literatura citada}

Aguayo-Lobo A., J. Urban-Ramírez y M. Salinas-Zacarías. 1984. El rorcual jorobado (Megaptera novaeangliae), durante la estación de reproducción en la bahía de Banderas México, y su distribución en el Pacífico mexicano. Memorias de la IX Reunión Internacional para el Estudio de los Mamíferos Marinos. Facultad de Ciencias, UNAM, México, D.F. 24 p.

Aguayo-Lobo A., S. Gaona, G. López-Ortega y M. SalinasZacarías. 1990. Mamíferos marinos dulceacuícolas, semiacuáticos y con tendencia al agua. In Atlas Nacional de México. Tomo II, sección IV. Instituto de Geografía, UNAM, México, D.F. p. 8-9

Aurioles-Gamboa D. 1993. Biodiversidad y estado actual de los mamíferos marinos en México. Revista de la Sociedad Mexicana de Historia Natural XLIV:397-412.

Aurioles-Gamboa D., F. Sinsel, C. Fox, E. Alvarado y O. Maravilla. 1983. Winter migration of subadult male California Sea Lions (Zalophus californianus) in the southern part of Baja California. Journal of Mammalogy 64:513-518.

Aurioles-Gamboa D. y M. García-Rivas. 2005. Zalophus californianus. In Los mamíferos silvestres de México, G. Ceballos y G. Oliva (eds.). Fondo de Cultura EconómicaCONABIO, México, D.F. p. 398-399.

Casas-Andreu, G. 1978. Análisis de la anidación de las tortugas marinas del género Lepidochelys en México. Anales del Centro de Ciencias del Mar y Limnología 5:141-158.

Connor, R. C., M. R. Heithaus, P. Berggren y J. L. Miksis. 2000. "Kerplunking": surface fluke-splashes during shallow-water bottom foraging by bottlenose dolphins. Marine Mammal Science 16:646-653.

Delgado, A. 1997. Relación de las toninas, Tursiups truncatus y las toninas moteadas, Stenella frontalis con la actividad camaronera en la sonda de Campeche, México. Anales del Instituto de Biología, Universidad Nacional Autónoma de México, Serie Zoología 68:317-338.

Dizon A., W. Perrin y P. Akin. 1994. Stocks of dolphins (Stenella $s p$. and Delphinus delphis) in the eastern tropical Pacific: a phyleogeographic classification. NOAA Technical Report NMFS 119:23 p.

Gallo-Reynoso, J. P. y A. Ortega-Ojeda. 1986. The first report of Zalophus Californianus in Acapulco, Mexico. Marine Mammal Science 2:158.

Gallo-Reynoso, J. P., A. Ortega-Ojeda y W. López. 1986. Humpback whales from México. Marine Mammal Science 2:78-79.

Gallo-Reynoso, J. P. y J. L. Solorzano-Velasco. 1991. Two new sightings of California Sea Lions on the Southern Coast of Mexico. Marine Mammal Science 9:96.

Gendron, D. 1993. Evidence of feeding by humpback whales (Megaptera novaeangliae) in the Baja California Breeding Ground, Mexico. Marine Mammal Science 9:76-81.

Hall, E. R. 1981. The mammals of North America, vol. 2. Wiley, New York. $1181 \mathrm{p}$.

Heyning, J. y M. Dahlheim. 1988. Orcinus orca. Mammalian Species 304:1-9.

Jefferson, T.A. y S. Leatherwood, 1995. Mamíferos marinos. In Guía FAO para la identificación de especies para los fines de pesca, Pacífico Centro-Oriental, vol. 3, W. Fischer, F. Krupp, W. Schneider, C. Sommer, K. E. Carpenter y V. H. Niem (eds.). FAO, Roma. p.1671 - 1745

Leatherwood, S., R. R. Reeves, F. W. Perrin y E. W. Evans. 1988. Ballenas, delfines y marsopas del pacifico nororiental y de las aguas árticas adyacentes. Comisión Interamericana del Atún Tropical. $245 \mathrm{p}$.

Márquez, R. 2000. Las tortugas marinas y nuestro tiempo, Segunda edición. La Ciencia para todos 144. Fondo de Cultura Económica, México, D.F. 197 p.

Meraz, J. 2003. Primer registro del lobo marino de California (Zalophus californianus) en Oaxaca, México. Ciencia y Mar 21:50-53.

Meraz, J. Y F. Becerril-Morales. 2004. Primer reporte de un 
ejemplar varado de Pseudorca crassidens en Zipolite, Oaxaca. Anales del instituto de Biología, Universidad Nacional Autónoma de México, Serie Zoología 75:229-235.

Nowak, R y J. Paradiso. 1983. Mammals of the world. The Johns Hopkins University Press, Baltimore, Maryland. 1362 p.

Odell, D. K. 1981. California sea lion Zalophus californianus (Lesson, 1828). In Handbook of marine mammals, S. H. Ridgway y R. Harrison (eds.). Academic Press, New York. p. 67-97

Odell, D. K. 1987. The mystery of marine mammal strandings. Cetus (Fall): 2-6.

Pachecho-Sandoval, P. 1991. Oceanografía física. In Oceanografía de los mares mexicanos. G. de la Lanza-Espino (ed.). AGT , México. p. 151-176.

Pérez-Bouchez, D y G. Gordillo-Morales. 2002. Avistamientos y primeros registros de varamientos de mamíferos marinos en las costas de Oaxaca (Huatulco-Puerto Escondido) de febrero de 1998 a mayo de 1999. Memorias de la XXVII Reunión Internacional para el Estudio de los Mamíferos Marinos, Veracruz, Veracruz. 59 p.

Perrin, W. 1998. Stenella longirostris. Mammalian Species
599:1-7.

Raymond, E. 1996. The mammals of North America.. Wiley, New York. 1075 p.

Salinas, M, y P. Ladrón de Guevara. 1993. Riqueza y diversidad de los mamíferos marinos. Ciencias 7:85-93.

Sánchez-Díaz, V. y J. Meraz. 2001. Registro de depredación sobre Dermochelys coriacea, en las costas de Oaxaca por Orcinus orca. Ciencia y Mar 5:51-54

SEMARNAP. 1998. Programa de manejo del Parque Nacional Huatulco. SEMARNAP. México, D.F. 225 p.

Steiger, G. H., J. Calambokidis, R. Sears, K. C. Balcomb y J. C. Cubbage. 1991. Movement of Humpback whales between California and Costa Rica. Marine Mammal Science 7:306310 .

Sutherland, W. 1983. Aggregation and the ideal free distribution. Journal of Animal Ecology 52:821-828.

Vázquez-Gutiérrez, F., S. López, A. Ramírez, M. Turner, F. Castillo y A. Valdés. 1998. La química del agua. In El golfo de Tehuantepec: el ecosistema y sus recursos, M. Tapia-García (ed.). Universidad Autónoma MetropolitanaIztapalapa, México, D.F. p. 35-50. 